\title{
Surgical Outcomes in Cancer Patients Undergoing Elective Surgery After Recovering from Mild-to-Moderate SARS-CoV-2 Infection
}

\author{
Anai N. Kothari, MD', Sandra R. DiBrito, $\mathrm{MD}^{1}$, J. Jack Lee, $\mathrm{PhD}^{2}$, Abigail S. Caudle, $\mathbf{M D}^{3}$, \\ Mark W. Clemens, $\mathrm{MD}^{4}$, Vijaya N. Gottumukkala, $\mathrm{MD}^{5}$, Matthew H. G. Katz, $\mathrm{MD}^{1}$, Anaeze C. Offodile, $\mathrm{MD}^{4}$, \\ Abhineet Uppal, $\mathrm{MD}^{6}$, , and George $\mathrm{J}^{\text {. Chang, } \mathrm{MD}^{6}}$ \\ ${ }^{1}$ Department of Surgical Oncology, University of Texas MD Anderson Cancer Center, Houston, TX; ${ }^{2}$ Department of \\ Biostatistics, University of Texas MD Anderson Cancer Center, Houston, TX; ${ }^{3}$ Department of Breast Surgical Oncology, \\ University of Texas MD Anderson Cancer Center, Houston, TX; ${ }^{4}$ Department of Plastic Surgery, University of Texas MD \\ Anderson Cancer Center, Houston, TX; ${ }^{5}$ Department of Anesthesia and Perioperative Medicine, University of Texas MD \\ Anderson Cancer Center, Houston, TX; ${ }^{6}$ Department of Colon and Rectal Surgery, University of Texas MD Anderson \\ Cancer Center, Houston, TX; ${ }^{7}$ Data-Driven Determinants for COVID-19 Oncology Discovery Effort (D3CODE) Team, \\ University of Texas MD Anderson Cancer Center, Houston, TX
}

\begin{abstract}
Background. An increasing number of patients with cancer diagnoses and prior SARS-CoV-2 infection will require surgical treatment. The objective of this study was to determine whether a history of SARS-CoV-2 infection increases the risk of adverse postoperative events following surgery in patients with cancer.

Methods. This was a propensity-matched cohort study from April 6, 2020 to October 31, 2020 at the UT MD Anderson Cancer Center. Cancer patients were identified who underwent elective surgery after recovering from SARS-CoV-2 infection and matched to controls based on patient, disease, and surgical factors. Primary study outcome was a composite of the following adverse postoperative events that occurred within 30 days of
\end{abstract}

Supplementary Information The online version contains supplementary material available at https://doi.org/10.1245/s10434021-10291-9.

(C) Society of Surgical Oncology 2021

First Received: 17 March 2021

Accepted: 27 May 2021;

Published Online: 26 June 2021

G. J. Chang, MD

e-mail: gchang@mdanderson.org surgery: death, unplanned readmission, pneumonia, cardiac injury, or thromboembolic event.

Results. A total of 5682 patients were included for study, and $114(2.0 \%)$ had a prior SARS-CoV-2 infection. The average time from infection to surgery was 52 (range 20-202) days. Compared with matched controls, there was no difference in the rate of adverse postoperative outcome (14.3\% vs. $13.4 \%, p=1.0)$. Patients with a SARS-CoV-2related inpatient admission before surgery had increased odds of postoperative complication (adjusted odds ratio [aOR] 7.4 [1.6-34.3], $p=0.01)$.

Conclusions. A minimal wait time of 20 days after recovering from minimally symptomatic SARS-CoV-2 infection appears to be safe for cancer patients undergoing low-risk elective surgery. Patients with SARS-CoV-2 infections requiring inpatient treatment were at increased risk for adverse events after surgery. Additional wait time may be required in those with more severe infections.

Patients with acute, perioperative SARS-CoV-2 infection are at increased risk of adverse events, including postoperative pulmonary complications and mortality. ${ }^{1}$ To mitigate these risks, several strategies have been implemented, including universal asymptomatic preoperative testing, risk prioritization, and COVID-19-free surgical pathways. ${ }^{2-5}$ These approaches attempt to manage risk by deferring elective surgery in patients with SARS-CoV-2 
infection and prioritizing conditions that require prompt treatment. ${ }^{6}$ For time-sensitive conditions, such as cancer, patients may require relatively urgent surgery after a SARS-CoV-2 infection, but the ideal interval for delay in surgery is unknown.

Additionally, the long-term physiologic sequelae associated with SARS-CoV-2 infection are becoming increasingly recognized. ${ }^{7}$ This "long-COVID" syndrome is not well understood, nor are the impacts of these prolonged physiologic insults on postoperative outcomes. ${ }^{8,9}$ Similar to other viral pulmonary syndromes that can impact outcomes after surgery, there may be a heightened risk of postoperative complications in those recently recovered from SARS-CoV-2 infection. This has prompted expert groups to propose safe wait periods to delay surgery after recovering from COVID-19 that range from 20 days to 8 weeks or longer. ${ }^{10}$ However, prolonged delays in care could cause more harm than good in cancer patients and are not yet evidence-based.

There is a paucity of data to guide decision-making on management of the SARS-CoV-2-recovered population requiring time-sensitive surgery, while at the same time, prevalence of patients who have recovered is increasing rapidly. Therefore, the primary objective of this study was to assess the postoperative outcomes of patients with cancer who underwent surgery after recovering from SARSCoV-2 infection. Secondary objectives included identifying risk factors for developing adverse postoperative events and evaluating the timing of surgery after infection recovery. In demonstrating the postoperative risk for this cohort and elucidating particular factors that put this population at risk, we can provide better care for this growing population.

\section{METHODS}

\section{Study Setting and Participants}

Cancer patients who underwent elective surgical procedures at the University of Texas MD Anderson Cancer Center between April 6, 2020 and October 31, 2020 were retrospectively reviewed to identify those with prior SARSCoV-2 infection. This included patients with both asymptomatic and symptomatic infections based on a positive SARS-CoV-2 PCR test any time before surgery. Institutional policy during the study period required at least 20 days between positive test and surgery with resolution of symptoms (fever-free without antipyretics, no cough/ shortness of breath/sore throat, no chills, no new loss of smell or taste, no muscle pain, no gastrointestinal symptoms, and no chest imaging suspicious for COVID-19). This wait period was determined using guidance from the
Centers for Disease Control and Prevention (CDC) and the observed duration of recovering replication-competent virus from patients with SARS-CoV-2. ${ }^{11,12}$ Patients recovered from SARS-CoV-2 were matched to controls who underwent the same operation during the same time period. Emergent surgeries were excluded (Fig. 1).

\section{Primary and Secondary Outcomes}

The primary outcome of study was composite SARSCoV-2-related adverse event defined as the development of postoperative pulmonary complication (pneumonia), postoperative cardiac injury (troponin elevation), thromboembolic event (acute pulmonary embolism or deep vein thrombosis), unplanned readmission, or death within 30 days of surgery. These outcomes were based on literature to date indicating these are common causes of morbidity related to SARS-CoV-2 infection. ${ }^{13,14}$ Secondary outcomes included postoperative length of stay, discharge disposition (home, home with home health, rehabilitation facility, death), postoperative oxygen saturation (overall and minimum), postoperative oxygen device use, postoperative hypoxemia, postoperative electrocardiogram order, and postoperative cardiac arrythmia.

\section{Data Source}

Electronic health data were obtained from structured and unstructured electronic health record elements, clinical note text, laboratory and pathology data, and perioperative sensor information within the Context Engine, MD Anderson's data management system. All data were integrated using Palantir Foundry (Syntropy), an interoperable and extensible cloud-based platform within the Context Engine that streamlines back-end data management and front-end data analysis, allowing rapid insight from multisourced data streams. ${ }^{15,16}$

\section{Statistical Approach}

Patients were classified as SARS-CoV-2-recovered if they had a confirmed COVID-19 infection at any time prior to elective surgery. Per institutional policy, all patients that underwent surgery also were required to have resolution of all SARS-CoV-2 symptoms as attested to during preoperative screening. For comparisons between recovered and control patients, univariable statistical testing was performed using two-sample t-tests and chi-squared tests.

SARS-CoV-2-recovered patients were matched 1:1 to SARS-CoV-2-negative patients using propensity scores calculated from a fitted multivariable logistic regression model. Matching variables were age, sex, race, comorbid disease, disease site, and type of surgery. Nearest neighbor 
FIG. 1 Study design with inclusions and exclusions to derive final analytic cohort

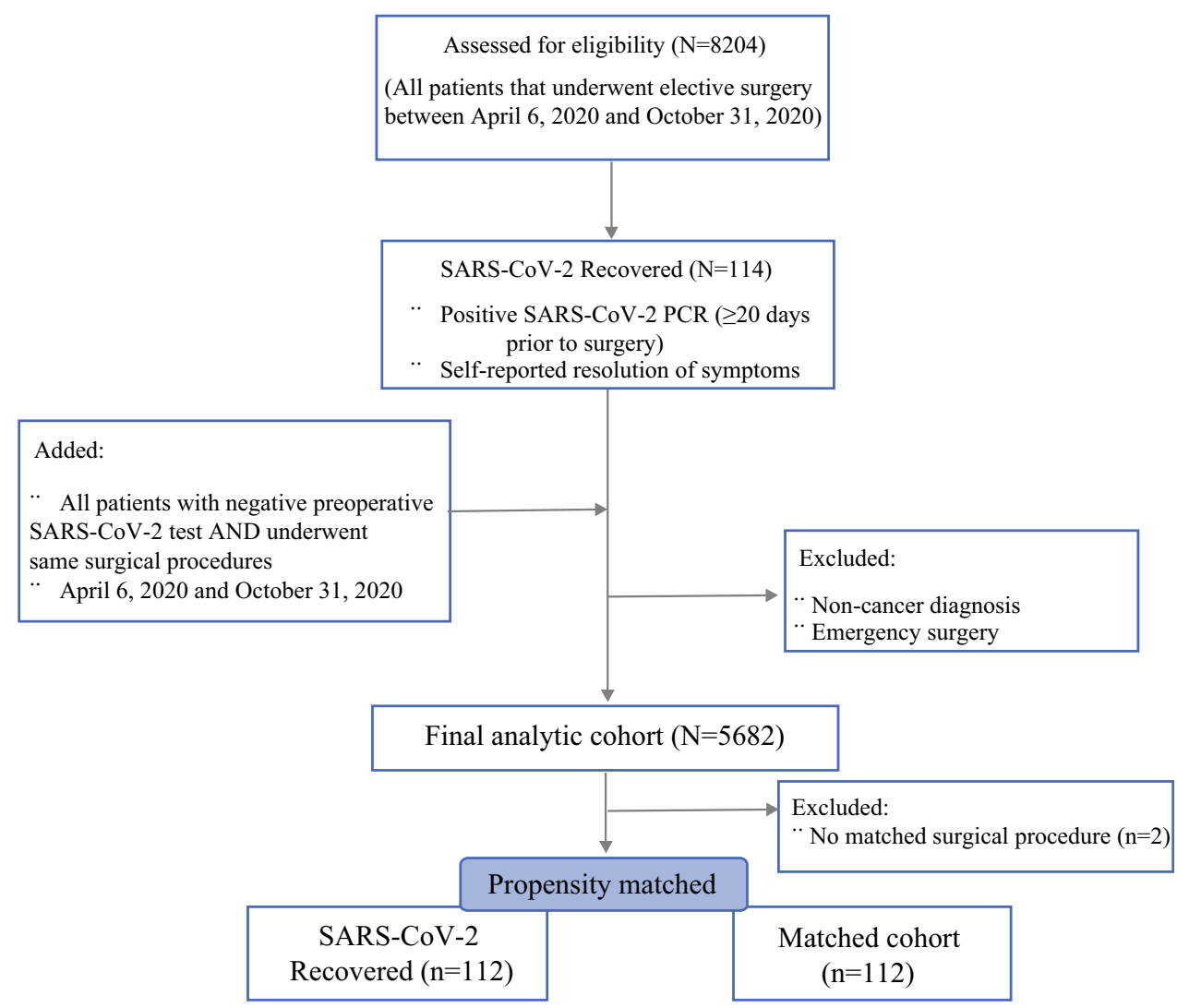

matching was conducted using the MatchIt package in $\mathrm{R}^{17}$ Match performance was assessed using absolute mean difference in covariables between groups. Continuous variables in matched groups were compared using paired $t$ tests and categorical variables using conditional logistic regression.

Logistic models were fit on SARS-CoV-2-recovered patients to investigate the association between preoperative factors and primary outcome. These included time from infection to surgery, preoperative SARS-CoV-2-related admission, type of surgery, age, sex, and Charlson Comorbidity Index. All analyses were performed using the Palantir Foundry platform.

\section{RESULTS}

In the cohort of 5682 patients included for study, 114 (2.0\%) had previously been infected with SARS-CoV-2 and recovered (Table 1). Of those, the average time from positive test result to surgery was 52 days (30 days standard deviation [SD]; range 20-202 days). This differed for patients with asymptomatic compared to symptomatic infections (47.1 vs. 66.4 days, $p \leq 0.01$; Fig. 2a). Over the course of the study period, the weekly percentage of patients undergoing surgery with a history of SARS-CoV-2 infection increased (Fig. 2b). Regarding the severity and treatment of SARS-CoV-2 infection, 8.8\% $(n=10)$ required inpatient admission at the time of their infection, $17.5 \%(n=20)$ received dexamethasone, $3.5 \%(n=4)$ received remdesivir, and $1.8 \%(n=2)$ received convalescent plasma.

When patients who had previously been infected with SARS-CoV-2 and recovered were matched with SARSCoV-2-negative patients, 112 matched pairs were formed. No difference in baseline characteristics were observed between matched groups (Table 2). Comparing SARSCoV-2-recovered patients undergoing surgery to propensity-matched controls, there was no statistically significant difference in length of stay (98 vs. 64 hours, $p=0.3$ ), discharge disposition to home ( $96 \%$ vs. $95 \%, p=0.5)$, 30 -day readmission $(8 \%$ vs. $9 \%, p=0.8)$, or mortality $(1 \%$ vs. $0 \%, p=0.5$; Table 3 ). There were no known nosocomial transmissions of SARS-CoV-2 related to the perioperative care of this population.

Intraoperatively, there were no differences in mean $\mathrm{FiO} 2$ (fraction of inspired oxygen) requirements (61.5\% vs. $60.7 \%, p=0.6$ ). Postoperatively, there were no differences in oxygen saturation measurements throughout the hospitalization (average $97 \%$ and minimum 93\% for both groups). Both populations had similar utilization of nasal cannula devices $(88.4 \%$ vs. $86 \%, p=0.7)$ and highflow/facemask devices (64\% vs. $60.7 \%, p=0.6)$. Only 
TABLE 1 Baseline characteristics of the overall study population $(N=5682)$

\begin{tabular}{|c|c|}
\hline Characteristic & All patients \\
\hline Age $(y r)$, mean (SD) & $58.1(14.4)$ \\
\hline \multicolumn{2}{|l|}{ Sex, $n(\%)$} \\
\hline Male & 2309 (40.6) \\
\hline Female & $3373(59.4)$ \\
\hline \multicolumn{2}{|l|}{ Race, $n(\%)$} \\
\hline White & $4358(76.7)$ \\
\hline Black & $593(10.4)$ \\
\hline Asian & $284(5.0)$ \\
\hline Other/declined & $447(7.9)$ \\
\hline \multicolumn{2}{|l|}{ Comorbid disease, $n(\%)$} \\
\hline COPD/asthma & $868(15.3)$ \\
\hline Congestive heart failure & $149(2.6)$ \\
\hline Dementia & $48(0.8)$ \\
\hline Diabetes mellitus & $1243(21.9)$ \\
\hline Depression/anxiety & $1975(34.8)$ \\
\hline Hypertension & $2979(52.4)$ \\
\hline Peripheral vascular disease & $176(3.1)$ \\
\hline Renal disease & $493(8.7)$ \\
\hline \multicolumn{2}{|l|}{ Disease site, $n(\%)$} \\
\hline Breast & $1163(20.5)$ \\
\hline Urologic & $1464(25.8)$ \\
\hline Gastrointestinal & $419(7.4)$ \\
\hline Gynecologic & $374(6.6)$ \\
\hline Plastics & $462(8.1)$ \\
\hline Neurosurgery & $221(3.9)$ \\
\hline Vascular & $186(3.3)$ \\
\hline Head and neck & $201(3.5)$ \\
\hline Other & $1192(21.0)$ \\
\hline \multicolumn{2}{|l|}{ Surgical classification, $n(\%)$} \\
\hline Same day $(<12 \mathrm{hr})$ & $2936(51.7)$ \\
\hline Extended recovery (12-48 hr) & $1355(23.8)$ \\
\hline Inpatient ( $>48 \mathrm{hr}$ ) & $1391(24.5)$ \\
\hline SARS-CoV-2-recovered $n(\%)$ & $114(2.0)$ \\
\hline
\end{tabular}

$29.5 \%$ of the SARS-CoV-2-recovered patients suffered postoperative hypoxemia (oxygen saturation $<93 \%$ ), which was not different from the matched controls $(27.7 \%, p=$ $0.7)$. Both groups had similar rates of pneumonia ( $4.5 \%$ vs. $5.4 \%, p=0.8$ ), and there was no difference in the rate of cardiac morbidity. Each group had a single patient with a thromboembolic event in the postoperative period (Table 3). A subgroup analysis based on complexity of surgery showed no differences (Supplementary Table 1).

In terms of COVID-19-specific postoperative complications, pneumonia, acute thromboembolic event, cardiac injury, and 30-day unplanned readmissions were considered as a composite outcome. Within the group of recovered SARS-CoV-2 patients, after controlling for age, sex, baseline comorbidity, type of surgery (same day, extended recovery, inpatient), and time from SARS-CoV-2 infection to surgery, inpatient admission for SARS-CoV-2 treatment was associated with higher odds of an adverse event (adjusted odds ratio [aOR] 7.35, 95\% confidence interval [CI] 1.58-34.28; Table 4).

\section{DISCUSSION}

In this study of SARS-CoV-2-recovered cancer patients and matched controls who underwent cancer-related surgery, we found no difference in length of stay, readmissions, or mortality. The recovery time from infection to surgery ranged from 20 days to 202 days. There was no significant difference in adverse pulmonary outcomes, acute VTE, cardiac events, 30-day readmissions, or death. Importantly, an inpatient admission for treatment of SARS$\mathrm{CoV}-2$ before surgery was associated with an increased risk of adverse outcomes.

It is our institutional policy to wait 20 days following a positive test for asymptomatic patients or for 20 days following complete resolution of symptoms before pursuing surgery. This minimum wait period is shorter than other proposed guidelines. ${ }^{4,10}$ For example, the American Society of Anesthesiologists and Anesthesia Patient Safety Foundation released a joint statement in December, 2020 suggesting a 4-week delay for asymptomatic patients, 6 weeks for symptomatic patients, 8-10 weeks for symptomatic patients requiring hospitalization or with diabetes or immunocompromised, and 12 weeks for patients admitted to an intensive care unit. ${ }^{18}$ Based on our results, a shorter wait period appears appropriate for outpatient, short stay, or low complexity inpatient procedures in patients with asymptomatic or minimally symptomatic infections. These data should be used to guide future guidelines. However, for patients recovering from severe/critical SARS-CoV-2 infection or undergoing higher complexity operations, additional data are required to make recommendations on an optimal wait period.

We noted a significantly increased risk of complications for recovered patients who required inpatient hospitalization for their SARS-CoV-2 infection. Hospitalization is a surrogate marker for disease severity and advanced baseline comorbidity, and it could be used to determine which patients could benefit from more intensive perioperative evaluations. It may be appropriate to prolong deferment of surgery in this population as well; however, more data are required to determine what an ideal interval would be for this group. Overall, patients who recovered did not have signs of worse cardiopulmonary function compared with 
(a)
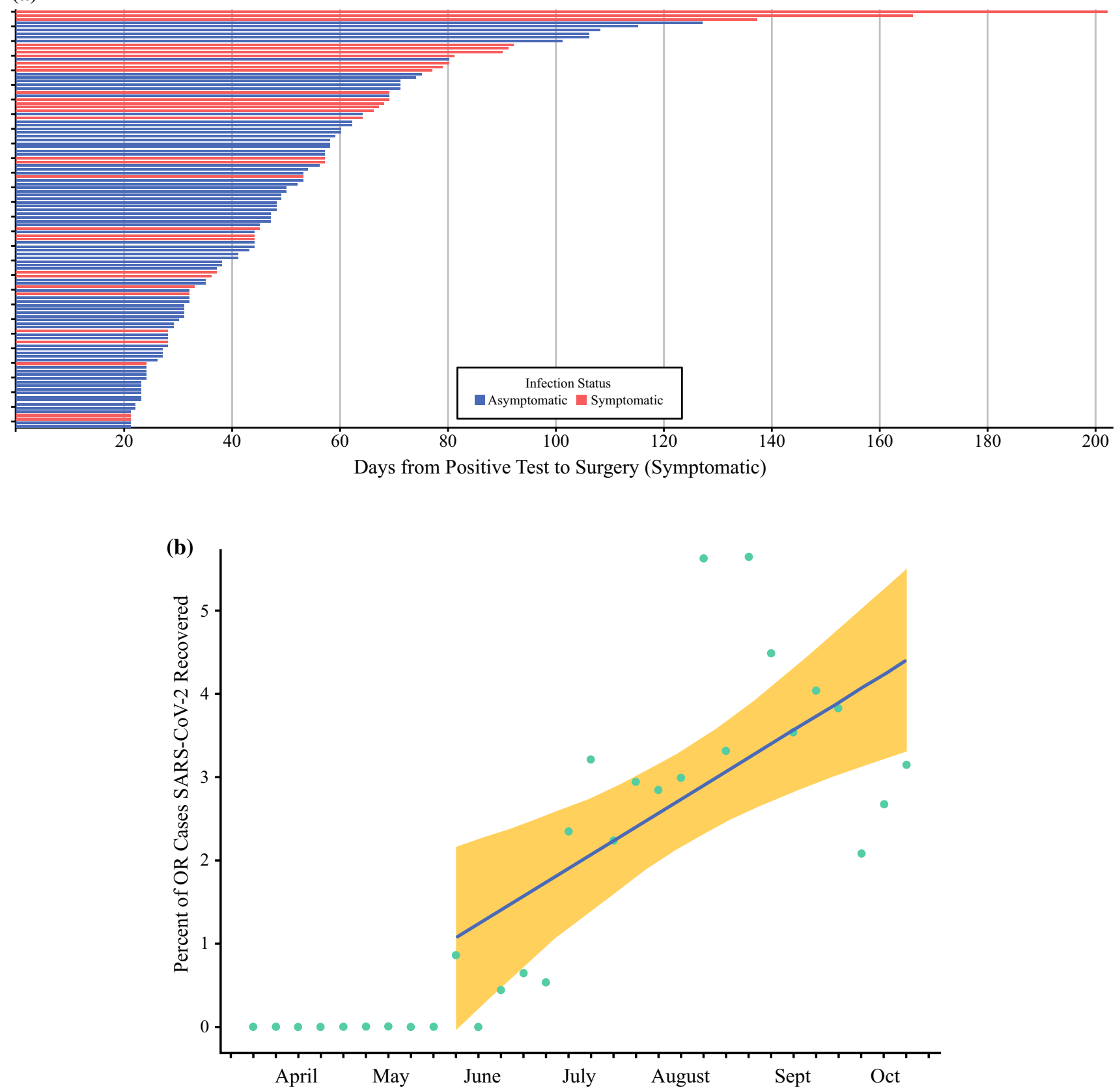

FIG. 2 a Time from SARS-CoV-2 diagnosis to surgery, stratified by patients with symptomatic or asymptomatic infection. b Percentage of surgical procedures performed in SARS-CoV-2-recovered patients, by week, from April 6, 2020-October 31, 2020

matched controls, as demonstrated by similarities in intraoperative and postoperative oxygen requirements, postoperative oxygen saturation levels, pneumonia, and cardiac event occurrences. This suggests that the majority of recovered patients do not require special preoperative cardiopulmonary evaluation and argues against universal preoperative SARS-CoV-2-recovered optimization programs. These resources may be better utilized if directed at those patients with more severe SARS-CoV-2 infection.
Given what is known about the SARS-CoV-2 infection, our study focused on outcomes that were more likely to be directly impacted by infection. ${ }^{13}$ While we demonstrated that there is no difference between recovered patients and matched controls in pneumonia, acute venous thromboembolism, cardiac injury, and readmission, there is additional work to be done to understand how other domains of surgical recovery are influenced. This is especially important as an increasing number of patients who have recovered from COVID-19 continue to report lasting 
TABLE 2 Baseline patient characteristics in matched groups $(\mathrm{n}=224)$

\begin{tabular}{|c|c|c|c|c|}
\hline Characteristic & SARS-CoV-2- recovered $(n=112)$ & $\begin{array}{l}\text { Matched cohort } \\
(n=112)\end{array}$ & $p$ value & Standardized absolute mean difference \\
\hline Age, mean (SD) & $51.7(15.8)$ & $54.3(17.1)$ & 0.2 & 0.18 \\
\hline \multicolumn{5}{|l|}{$\operatorname{Sex}$} \\
\hline Male & $40(35.7 \%)$ & $43(38.4 \%)$ & \multirow[t]{2}{*}{0.8} & 0.03 \\
\hline Female & $72(64.3 \%)$ & $69(61.6 \%)$ & & 0.03 \\
\hline \multicolumn{5}{|l|}{ Race } \\
\hline White & $69(61.6 \%)$ & $69(61.6 \%)$ & \multirow[t]{4}{*}{0.9} & $<0.01$ \\
\hline Black & $23(20.5 \%)$ & $24(21.4 \%)$ & & 0.01 \\
\hline Asian & $7(6.3 \%)$ & $7(6.3 \%)$ & & $<0.01$ \\
\hline Other/declined & $13(11.6 \%)$ & $12(10.7 \%)$ & & 0.02 \\
\hline \multicolumn{5}{|l|}{ Comorbid disease } \\
\hline COPD/asthma & $16(14.3 \%)$ & $22(19.6 \%)$ & 0.4 & 0.05 \\
\hline Congestive heart failure & $4(3.6 \%)$ & $3(2.7 \%)$ & 1.0 & 0.01 \\
\hline Diabetes mellitus & $29(25.9 \%)$ & $28(25.0 \%)$ & 1.0 & 0.01 \\
\hline Depression/anxiety & $49(43.8 \%)$ & $57(50.9 \%)$ & 0.3 & 0.07 \\
\hline Hypertension & $59(52.7 \%)$ & $73(65.2 \%)$ & 0.077 & 0.13 \\
\hline Peripheral vascular disease & $4(3.6 \%)$ & $3(2.7 \%)$ & 1.0 & 0.01 \\
\hline Renal disease & $13(11.6 \%)$ & $14(12.5 \%)$ & 1.0 & 0.01 \\
\hline \multicolumn{5}{|l|}{ Disease site } \\
\hline Breast & $23(20.5 \%)$ & $30(26.8 \%)$ & \multirow[t]{10}{*}{0.9} & 0.02 \\
\hline Urologic & $21(18.8 \%)$ & $21(18.8 \%)$ & & 0.01 \\
\hline Gastrointestinal & $15(13.4 \%)$ & $15(13.4 \%)$ & & 0.01 \\
\hline Gynecologic & $11(9.8 \%)$ & $7(6.2 \%)$ & & 0.04 \\
\hline Plastics & $8(7.1 \%)$ & $3(2.7 \%)$ & & 0.04 \\
\hline Neurosurgery & $9(8.0 \%)$ & $11(9.8 \%)$ & & 0.02 \\
\hline Orthopedic oncology & $5(4.5 \%)$ & $5(4.5 \%)$ & & $<0.01$ \\
\hline Endocrine & $4(3.6 \%)$ & $5(4.5 \%)$ & & 0.01 \\
\hline Head and neck & $5(4.5 \%)$ & $6(5.4 \%)$ & & 0.01 \\
\hline Other & $11(9.8 \%)$ & $9(8.0 \%)$ & & $<0.01$ \\
\hline \multicolumn{5}{|l|}{ Surgical classification } \\
\hline Same day $(<12 \mathrm{hr})$ & $49(43.8 \%)$ & $44(39.3 \%)$ & \multirow[t]{3}{*}{0.5} & 0.03 \\
\hline Extended recovery (12-48 hr) & $28(25.0 \%)$ & $36(32.1 \%)$ & & 0.02 \\
\hline Inpatient ( $>48 \mathrm{hr}$ ) & $35(31.2 \%)$ & $32(28.6 \%)$ & & 0.03 \\
\hline
\end{tabular}

$\%$ reduction bias $=99.3 \%$; mean bias $=<1.0 \%$

symptoms across organ systems that last longer than 12 weeks from onset. This includes persistent fatigue, respiratory symptoms, joint pain, cardiac manifestations, neurocognitive difficulties, gastrointestinal changes, and skin changes. ${ }^{8,19}$ Further study examining a larger cohort of SARS-CoV-2-recovered patients with persistent symptoms ("long COVID") will be necessary to more comprehensively understand the interplay between COVID-19 recovery and surgical outcomes.

There also are other important limitations to be considered when interpreting this study. All patients in the study had cancer diagnoses, making their care particularly time sensitive. They are closely followed and cared for at a highly specialized center; therefore, our results may not be generalizable to a broader surgical population. All emergency surgeries were excluded, and these data can only be applied to elective operations. Some patients in our cohort may have had a prior SARS-CoV-2 infection diagnosed and treated at another facility. Although all new patients are asked to provide this information, it is possible not all preoperative infections are captured in this study. In order to study a sufficiently large group, the study population included patients undergoing different surgical procedures at different disease sites, with the majority being outpatient 
TABLE 3 Perioperative surgical outcomes for patients undergoing surgery after recovering from SARS-CoV-2 compared with matched controls

\begin{tabular}{|c|c|c|c|}
\hline Characteristic & SARS-CoV-2- recovered $(N=112)$ & $\begin{array}{l}\text { Matched cohort } \\
(N=112)\end{array}$ & $p$ value \\
\hline Composite adverse event, $n(\%)$ & $16(14.3)$ & $15(13.4)$ & 1.0 \\
\hline Length of stay, mean hours (SD) & $97.8(288.0)$ & $63.7(140.0)$ & 0.3 \\
\hline Readmission at 30 days, $n(\%)$ & $9(8.0)$ & $10(8.9)$ & 0.8 \\
\hline \multicolumn{4}{|l|}{ Discharge disposition, $n(\%)$} \\
\hline Home & $108(96.4)$ & $106(94.6)$ & 0.5 \\
\hline Home with home health & $2(1.8)$ & $5(4.5)$ & \\
\hline Rehabilitation facility & $1(0.9)$ & $1(0.9)$ & \\
\hline Death & $1(0.9)$ & $0(0.0)$ & \\
\hline \multicolumn{4}{|l|}{ Intraoperative } \\
\hline $\mathrm{FiO}_{2}$ (mean) & $61.5 \%$ & $60.7 \%$ & 0.6 \\
\hline \multicolumn{4}{|l|}{ Pulmonary } \\
\hline Postoperative oxygen saturation (average \%) & $97.4 \%$ & $97.4 \%$ & 0.7 \\
\hline Postoperative oxygen saturation (min \%) & $93.0 \%$ & $93.1 \%$ & 0.9 \\
\hline Postoperative nasal cannula use, $n(\%)$ & $99(88.4)$ & 97 (86.6) & 0.7 \\
\hline Postoperative high flow or facemask, $n(\%)$ & $72(64.3)$ & $68(60.7)$ & 0.6 \\
\hline Postoperative hypoxemia $\left(\mathrm{SpO}_{2}<93 \%\right), n(\%)$ & $33(29.5)$ & $31(27.7)$ & 0.8 \\
\hline Pneumonia, $n(\%)$ & $5(4.5)$ & $6(5.4)$ & 0.8 \\
\hline \multicolumn{4}{|l|}{ Cardiac, $n(\%)$} \\
\hline Postoperative myocardial injury (elevated troponin) & $4(3.6)$ & $1(0.9)$ & 0.4 \\
\hline Postoperative cardiac arrhythmia & $5(4.5)$ & $5(4.5)$ & 1.0 \\
\hline Postoperative EKG ordered & $4(3.6)$ & $2(1.8)$ & 0.7 \\
\hline \multicolumn{4}{|l|}{ Thromboembolic, $n(\%)$} \\
\hline DVT or PE & $1(0.9)$ & $1(0.9)$ & 1.0 \\
\hline
\end{tabular}

TABLE 4 Risk factors for composite COVID-specific postoperative complication (pneumonia, acute thromboembolic event, cardiac injury, 30-day readmission) in patients recovered from SARS$\mathrm{CoV}-2$ infection

\begin{tabular}{|c|c|c|c|c|}
\hline & \multicolumn{2}{|l|}{ Model $\mathrm{A}^{1}$} & \multicolumn{2}{|l|}{ Model $\mathrm{B}^{2}$} \\
\hline & Odds ratio $(95 \% \mathrm{CI})$ & $p$ & Odds ratio $(95 \% \mathrm{CI})$ & $p$ \\
\hline SARS-CoV-2 inpatient admission & $7.35(1.58-34.28)$ & 0.01 & $6.39(1.45-29.14)$ & 0.01 \\
\hline Age & $0.99(0.94-1.05)$ & 0.79 & - & - \\
\hline Gender (M) & $0.82(0.24-2.79)$ & 0.75 & - & - \\
\hline Charlson index & $0.94(0.82-1.08)$ & 0.38 & - & - \\
\hline Weeks from infection & $0.71(0.40-1.26)$ & 0.25 & - & - \\
\hline \multicolumn{5}{|l|}{ Surgery classification } \\
\hline Same day & Ref & & Ref & \\
\hline Extended recovery & $2.05(0.42-10.02)$ & 0.37 & & \\
\hline Inpatient & $4.04(1.01-16.21)$ & 0.05 & $3.13(0.99-9.9)$ & 0.05 \\
\hline
\end{tabular}

${ }^{1}$ Model A: Demographic and surgical variables included to further explore the association between inpatient surgical stay and composite outcome

${ }^{2}$ Model B: Includes only significant variables from Model A and transforms surgical classification into binary variable or short-stay procedures. While we attempted to address the heterogeneity of the study population by identifying a control group that underwent similar procedures and used propensity score matching to adjust for measured confounders, our findings must be interpreted in the context of these limitations. Importantly, patient selection and provider decision-making on how long to defer surgery 
could not be accounted for. While 20 days is the minimal required wait period at our institution, our data demonstrate a wide range in the timing between infection and delay.

As the COVID-19 pandemic continues, there will undoubtedly be more patients with preexisting SARS-CoV2 infection requiring surgery for a variety of indications. Our understanding of this infection, both short-term and long-term sequelae, is evolving. It is imperative that we rapidly study and share knowledge to improve outcomes of patients in real time. Based on our series, a minimum wait time of 20 days appears to be safe for cancer patients undergoing elective outpatient or short-stay surgery after recovering from mild to moderate SARS-CoV-2. For patients recovering from severe SARS-CoV-2 infection that required inpatient hospitalization, an increased risk of adverse events was observed and additional efforts for preoperative evaluation, optimization, and postoperative monitoring should be considered in this population.

ACKNOWLEDGMENT The authors would like to especially acknowledge the contribution of Andrew T. Trans, Mark Knafl, and Benju Vicknamparampil, members of the D3CODE Team at MD Anderson. This work is supported in part by the National Cancer Institute of the National Institutes of Health under Award Number CA016672 (The University of Texas MD Anderson Cancer Center Support Grant).

\section{REFERENCES}

1. Nepogodiev D, Bhangu A, Glasbey JC, et al. Mortality and pulmonary complications in patients undergoing surgery with perioperative SARS-CoV-2 infection: an international cohort study. Lancet. 2020;396(10243):27-38.

2. Tzeng C-WD, Cao HST, Roland CL, et al. Surgical decisionmaking and prioritization for cancer patients at the onset of the COVID-19 pandemic: a multidisciplinary approach. Surg Oncol. 2020;34:182-5.

3. Boffa DJ, Judson BL, Billingsley KG, et al. Results of COVIDminimal surgical pathway during surge-phase of COVID-19 pandemic. Ann Surg. 2020;272(6):e316-20.

4. Baiocchi G, Aguiar S Jr, Duprat JP, et al. Early postoperative outcomes among patients with delayed surgeries after preoperative positive test for SARS-CoV-2: a case-control study from a single institution. J Surg Oncol. 2021. https://doi.org/10.1002/jso. 26377.
5. Kothari AN, Trans AT, Caudle AS, et al. Universal preoperative SARS-CoV-2 testing can facilitate safe surgical treatment during local COVID-19 surges. Br J Surg. 2020. https://doi.org/10.109 3/bjs/znaa062.

6. Myles PS, Maswime S. Mitigating the risks of surgery during the COVID-19 pandemic. Lancet. 2020;396(10243):2-3.

7. Wise J. Long covid: doctors call for research and surveillance to capture disease. BMJ. 2020;370:m3586.

8. Mahase E. Covid-19: what do we know about "long covid"? BMJ. 2020;370:m2815.

9. Mandal S, Barnett J, Brill SE, et al. "Long-COVID": a crosssectional study of persisting symptoms, biomarker and imaging abnormalities following hospitalisation for COVID-19. Thorax. 2020. https://doi.org/10.1136/thoraxjnl-2020-215818.

10. COVID-19 and Elective Surgery. Accessed 18 Jan 2021. Available at: https://www.asahq.org/in-the-spotlight/coronavirus-c ovid-19-information/elective-surgery.

11. van Kampen JJA, van de Vijver DAMC, Fraaij PLA, et al. Duration and key determinants of infectious virus shedding in hospitalized patients with coronavirus disease-2019 (COVID-19). Nat Commun. 2021;12(1):267.

12. Aydillo T, Gonzalez-Reiche AS, Aslam S, et al. Shedding of viable SARS-CoV-2 after immunosuppressive therapy for cancer. $N$ Engl J Med. 2020;383(26):2586-8.

13. Guan W-J, Ni Z-Y, Hu Y, et al. Clinical characteristics of coronavirus disease 2019 in China. $N$ Engl J Med. 2020;382(18):1708-20.

14. Donnelly JP, Wang XQ, Iwashyna TJ, Prescott HC. Readmission and death after initial hospital discharge among patients with COVID-19 in a large multihospital system. JAMA. 2020. https://d oi.org/10.1001/jama.2020.21465.

15. Palantir Foundry. Accessed 9 Jan 2021. Available at: https://w ww.palantir.com/palantir-foundry/

16. Syntropy. Accessed 21 Feb 2021. Available at: https://www.syn tropy.com/news/

17. Ho DE, Imai K, King G, Stuart EA. MatchIt: nonparametric preprocessing for parametric causal inference. J Stat Softw. 2011. https://doi.org/10.18637/jss.v042.i08.

18. ASA and APSF Joint Statement on elective surgery and anesthesia for patients after COVID-19 infection. Accessed 18 Jan 2021. Available at: https://www.asahq.org/about-asa/newsroom/ news-releases/2020/12/asa-and-apsf-joint-statement-on-elective-s urgery-and-anesthesia-for-patients-after-covid-19-infection.

19. Greenhalgh T, Knight M, A'Court C, Buxton M, Husain L. Management of post-acute COVID-19 in primary care. BMJ. 2020;370:m3026.

Publisher's Note Springer Nature remains neutral with regard to jurisdictional claims in published maps and institutional affiliations. 